\title{
Clinical Features of a New Disease Concept, IgG4-related Thyroiditis
}

Category submitted : Article

Short title: IgG4-related thyroiditis

Takayuki Watanabe, ${ }^{1}$ Masahiro Maruyama, ${ }^{1}$ Tetsuya Ito, ${ }^{1}$ Yasunari Fujinaga, ${ }^{2}$ Yayoi Ozaki, ${ }^{1}$ Masafumi Maruyama, ${ }^{1}$ Ryou Kodama, ${ }^{1}$ Takashi Muraki, ${ }^{1}$ Hideaki Hamano, ${ }^{3}$ Norikazu Arakura, ${ }^{4}$ Masumi Kadoya, ${ }^{2}$ Satoru Suzuki, ${ }^{5}$ Mitsuhisa Komatsu, ${ }^{5}$ Hisashi Shimojo, ${ }^{6}$ Kenji Notohara, ${ }^{7}$ Mitsuo Uchida, ${ }^{8}$ and Shigeyuki Kawa ${ }^{8}$

${ }^{1}$ Department of Gastroenterology, ${ }^{2}$ Department of Radiology, ${ }^{3}$ Department of Medical Information, ${ }^{4}$ Endoscopic Examination Center, ${ }^{5}$ Department of Aging Medicine and Geriatrics, and ${ }^{6}$ Department of Pathology, Shinshu University School of Medicine, 3-1-1 Asahi, Matsumoto 390-8621, Japan

${ }^{7}$ Department of Pathology, Kurashiki Central Hospital, 1-1-1 Miwa, Kurashiki 710-8602, Japan

${ }^{8}$ Center for Health, Safety, and Environmental Management, Shinshu University, 3-1-1 
Asahi, Matsumoto 390-8621, Japan

Corresponding author: Shigeyuki Kawa

Center for Health, Safety, and Environmental Management, Shinshu University, 3-1-1

Asahi, Matsumoto 390-8621, Japan

E-mail: skawapc@ shinsyu-u.ac.jp, Phone: +81-263-37-2157, Fax: +81-263-37-2183

\section{Abstract}

\section{Objectives}

IgG4-related disease is a recently proposed systemic disorder that includes autoimmune pancreatitis (AIP), Mikulicz's disease, and various other organ lesions. In the present study, we examined whether thyroid lesion should also be considered as IgG4-related disease under the new term IgG4-related thyroiditis.

\section{Methods}

We enrolled 114 patients with IgG4-related disease, including 92 patients with AIP, 15

patients with Mikulicz's disease, and 7 patients with IgG4-related cholangitis, and analyzed clinical findings, thyroid function, serum values of activity markers, CT 
images and histology of the thyroid gland.

\section{Results and Conclusion}

Among the 22 patients (19\%) in our cohort who were found to have hypothyroidism (TSH $>4 \mathrm{mIU} / \mathrm{L}), 11$ patients had clinical hypothyroidism $(\mathrm{FT} 4<1 \mathrm{ng} / \mathrm{dl})$ and 11 patients had subclinical hypothyroidism (FT4 $\geqq 1 \mathrm{ng} / \mathrm{dl}$ ). Serum concentrations of IgG, IgG4, circulating immune complex, and $\beta 2$ microglobulin were significantly higher in the hypothyroidism group compared with the remaining 92 euthyroid patients, and that of C3 was significantly lower. After prednisolone treatment, TSH values had significantly decreased $(\mathrm{P}=0.005)$ and $\mathrm{T} 4$ values had significantly increased $(\mathrm{P}=0.047)$ in patients with hypothyroidism. CT images showed that the thyroid gland of clinical hypothyroidism had a significantly greater volume than those of euthyroid and other groups. Pathological analysis of one resected thyroid gland disclosed a focused lesion with infiltration of lymphocytes and IgG4-bearing plasma cells and loss of thyroid follicles.

In conclusion, thyroid lesion showing hypothyroidism appears to be a new disease termed IgG4-related thyroiditis. Awareness of this condition will lead to appropriate 
corticosteroid treatment that may prevent progression to a fibrous state.

Keywords: IgG4, IgG4-related disease, IgG4-related thyroiditis, Hashimoto's thyroiditis,

hypothyroidism 


\section{Introduction}

Autoimmune pancreatitis (AIP) is a specific type of chronic pancreatitis caused in part by autoimmune mechanisms.[1, 2] The characteristic features of AIP are high serum IgG4 concentration, IgG4-bearing plasma cell infiltration, and responsiveness to steroid therapy, all of which are useful for diagnosis and evaluation of disease activity.[3, 4] AIP has been reported to be complicated with various extra-pancreatic lesions, such as sclerosing cholangitis, lachrymal gland inflammation, sialoadenitis, and retroperitoneal fibrosis.[5-7] These involvements share the common features of abundant IgG4-bearing plasma cell infiltration and favorable response to corticosteroid therapy, suggesting the presence of a common pathogenic background. Recently, the concept of a comprehensive systemic disease associated with IgG4, termed IgG4-related disease, has been proposed for these lesions[8][8], in which AIP has been regarded as a pancreatic manifestation.[9] The characteristic features of IgG4-related disease have been identified as 1) high serum IgG4 concentration, 2) serum changes of a variety of activity markers, including elevated circulating immune complex (CIC), elevated soluble IL2 receptor (sIL2R), and low C3 and C4 levels, 3) image findings of organ 
enlargement, mass lesion, and wall thickening, 4) favorable response to corticosteroid therapy, 5) abundant IgG4-bearing plasma cell infiltration in affected organs, and 6) complication with other IgG4-related diseases.

Mounting evidence is suggesting that IgG4-related disease includes thyroid lesion as well. Multifocal idiopathic fibrosclerosis (MIF) is a systemic disease $[10,11]$ whose distribution is very similar to that of IgG4-related disease and is thought to be the same entity.[12, 13] Since MIF involves Riedel's thyroiditis, it is possible that IgG4-related disease includes thyroid lesion; in pathological studies, Riedel's thyroiditis was reported to be a manifestation of IgG4-related disease,[14] and a subtype of Hashimoto's thyroiditis was also found to be involved in this condition due to abundant IgG4-bearing plasma cell infiltration in thyroid tissue.[15] Furthermore, we previously reported that AIP was significantly associated with hypothyroidism, which was later confirmed by other studies. $[5,16,17]$

A correlation between IgG4-related disease and thyroid lesion has not yet been fully accepted as several common clinical features of Riedel's thyroiditis and Hashimoto's thyroiditis, such as severe fibrosis of the thyroid gland and diffuse goiter, 
are not typically seen in IgG4-related disease. [16] Thus, investigation of the characteristic features of IgG4-related disease, such as high prevalence of hypothyroidism, close association with a variety of serum activity markers, image findings of organ swelling or mass lesion, favorable response to corticosteroid therapy, and pathological findings of tissue injury and IgG4-bearing plasma cell infiltration, are needed. In the present study, we examined these parameters to verify whether thyroid lesion is in fact an IgG4-related disease and propose the concept of IgG4-related thyroiditis as a new disease entity.

\section{Materials and Methods}

\section{Patients}

A total of 114 patients with IgG4-related disease ( 89 male, 25 female; median age: 69 years, range: 38-85 years) who had been examined and treated at Shinshu University Hospital during the period from 1992 to 2011 were enrolled in this study. Our cohort consisted of patients with the following initial diagnosis: 92 patients with AIP (72 male, 20 female; median age: 66 years, range: 38-85 years), 15 patients with 
Mikulicz's disease (11 male, 4 female; median age: 70 years, range: 43-82 years) who were not complicated with AIP, and 7 patients with IgG4-related sclerosing cholangitis (6 male, 1 female; median age: 69 years, range: 58-84 years) who were not complicated with AIP or Mikulicz's disease. Diagnosis of AIP and Mikulicz's disease was based on the Asian diagnostic criteria for AIP [18] and the diagnostic criteria for IgG4-related Mikulicz's disease,[19] respectively. Diagnosis of IgG4-related sclerosing cholangitis was based on the diagnostic criteria for IgG4-associated cholangitis,[20] in which the patient showed cholangiography of intra/extra hepatic bile duct strictures similar to primary sclerosing cholangitis and high serum IgG4 concentration $(>135 \mathrm{mg} / \mathrm{dl})$ or abundant bile duct tissue infiltration with lymphoplasmacyte/IgG4-positive plasma cells. All cases also fulfilled comprehensive diagnostic criteria for IgG4-related disease, 2011, proposed by Japanese researchers.[21]

Since most patients had multiple lesions associated with IgG4-related disease, our cohort was also classifiable by type of IgG4-related disease as 92 patients with AIP, 41 patients with Mikulicz's disease, 71 patients with IgG4-related sclerosing cholangitis, 27 patients with retroperitoneal fibrosis, and 18 patients with kidney lesion. 
Efforts were made to exclude all patients with possible malignancies.

\section{Clinical and serological analysis}

We measured serum levels of free $\mathrm{T} 3$, free $\mathrm{T} 4$, thyroid stimulating hormone (TSH), anti-thyroglobulin (anti-TG) antibody, and anti-thyroid peroxidase (anti-TPO) antibody. We defined hypothyroidism as elevated TSH (>4 mIU/L), and further classified these subjects into the clinical hypothyroidism group with low free T4 $(<1 \mathrm{ng} / \mathrm{dL})$ and the subclinical hypothyroidism group with normal free $\mathrm{T} 4(\geq 1 \mathrm{ng} / \mathrm{dL})$. In addition to routine blood tests that included an assay for antinuclear antibody (ANA), we measured the serum concentration of a variety of activity markers, including $\operatorname{IgG} 4$, circulating immune complexes (CIC), soluble interleukin-2 receptor (sIL2R), $\beta 2$-microglobulin ( $\beta 2-\mathrm{MG})$, and complement $\mathrm{C} 3$ and $\mathrm{C} 4$, all of which have been considered as representative of IgG4-related disease activity.[3, 22]

\section{Imaging analysis}

We evaluated and compared thyroid CT images of IgG4-related disease among 20 euthyroid patients, 7 patients with subclinical hypothyroidism, and 5 patients with clinical hypothyroidism in whom we could assess thyroid volume and density. We also 
examined thyroid CT images of 24 normal control subjects over 60 years of age who were free of thyroid disease and had undergone CT imaging during the same period. We traced the transverse CT images of each thyroid gland using $2.5 \mathrm{~mm}$-thick slices. The areas of traced thyroid glands $\left(\mathrm{cm}^{2}\right)$ were calculated automatically by EV Insite software (PSP Co.Ltd, Tokyo). Thyroid volumes $\left(\mathrm{cm}^{3}\right)$ represented the sum of each thyroid gland area slice. The CT density (HU) represented the average density of each thyroid gland slice.

\section{Pathological analysis}

We examined the thyroid gland of a 63-year-old patient with IgG4-related disease as well as papillary thyroid carcinoma. The woman had Mikulicz's disease, AIP, and a serum IgG4 level of $1500 \mathrm{mg} / \mathrm{dl}$, and showed normal thyroid function before resection. Non-cancerous tissue samples were examined to check for the presence of tissue injury, lymphoplasmacytic infiltration, and IgG4-bearing plasma cells. IgG4 immunostaining was performed with anti-IgG4 antibody (AU009, The Binding Site, Birmingham, UK) and HRP-labeled rabbit anti-sheep immunoglobulin (p0163, DakoCytomation, Glostrup, Denmark).[4] 


\section{Statistical analysis}

Statistical analysis of clinical and serological studies was performed using Fisher's exact test and the Mann-Whitney U-test. Statistical analysis of imaging analysis was carried out using one-way analysis of variance with Tukey's HSD test or the Kruskal-Wallis test with the Bonferroni method. Analysis of variance among the 4 study groups and other comparisons were performed after Shapiro-Wilk testing of variance homogeneity. Differences with $\mathrm{p}$ values of $<0.05$ were considered to be statistically significant.

\section{Ethics}

This study was approved by the ethics committee of Shinshu University Hospital (approval number 1804).

\section{Results}

Frequency of hypothyroidism in IgG4-related disease

Among the 114 patients with IgG4-related disease, 22 (19\%) had hypothyroidism (TSH $>4 \mathrm{mIU} / \mathrm{L})$, which included 11 patients with clinical hypothyroidism (FT4<1ng/dl) 
and 11 patients with subclinical hypothyroidism (FT4 $\geq 1 \mathrm{ng} / \mathrm{dl}$ ) (figure 1). Two patients

who required levothyroxine supplementation were present in the clinical hypothyroidism group. A male preponderance was found at comparable levels in the hypothyroidism, and euthyroid state groups (table 1). The prevalence of either anti-TG antibody or anti-TPO antibody in the hypothyroidism group was $50 \%$, meaning half of these patients were negative for thyroid autoantibodies (table 1). No patients with hypothyroidism showed marked goiter or invasion of surrounding organs by severe fibrosis of the thyroid gland by physical examination, ultrasonography, or CT (data not shown).

\section{Comparison of hypothyroid and euthyroid patients}

Serum concentrations of IgG, IgG4, CIC, and $\beta 2 \mathrm{MG}$ were significantly higher in the 22 patients with hypothyroidism compared with the 92 euthyroid patients. Serum C3 was significantly lower in the hypothyroidism group (table 1). The frequency of anti-TG antibody was significantly greater in patients with hypothyroidism. There were no significant differences in the frequency of anti-TPO antibody or ANA between the two groups. The number of patients who had two or more IgG4-related lesions in the 
hypothyroid group was comparable to that in the euthyroid state group ( $82 \%$ vs. $74 \%$ ).

\section{Effects of prednisolone treatment on hypothyroid status}

We could evaluate the effects of prednisolone treatment in 10 patients with hypothyroidism on whom serial thyroid function tests were performed before and after corticosteroid treatment. After therapy, TSH values had significantly decreased $(\mathrm{P}=0.005)$ (figure $2 \mathrm{a})$ and $\mathrm{T} 4$ values had significantly increased $(\mathrm{P}=0.047)$ (figure $2 \mathrm{~b})$ in these patients, which were indicative of a favorable response.

\section{Comparison of clinical and subclinical hypothyroidism patients}

There were no significant differences in serum activity markers or frequencies of thyroid autoantibodies between patients with clinical hypothyroidism and those with subclinical hypothyroidism (data not shown).

\section{Imaging analysis}

CT images showed that the thyroid gland of clinical hypothyroidism was significantly greater in volume than those of normal subjects $(\mathrm{p}=0.002)$, euthyroid patients $(\mathrm{p}=0.005)$, and patients with subclinical hypothyroidism $(\mathrm{p}=0.003)$ by Tukey's HSD test (figure 3a). There was a significant difference in thyroid gland density among 
the 4 groups as a whole by the Kruskal-Wallis test, but none were seen between any pair using the Mann-Whitney U-test. The thyroid gland of clinical hypothyroidism tended to be less dense than those of the other 3 groups (figure $3 b$ ).

Serial changes of thyroid CT images in one patient with hypothyroidism demonstrated progression of atrophy and density loss in spite of thyroid function improvement after treatment, from $12.4 \mathrm{~cm}^{3}$ and $84 \mathrm{HU}$, respectively, at diagnosis, to 5.8 $\mathrm{cm}^{3}$ and 53HU 6 years later, to $4.7 \mathrm{~cm}^{3}$ and 54HU 7 years later (figure 4).

\section{Pathological analysis}

Pathological analysis of non-cancerous thyroid tissue samples from a patient with IgG4-related disease complicated by thyroid cancer revealed prominent infiltration of lymphocytes and IgG4-bearing plasma cells and loss of thyroid follicles (figure 5). The focus was small and non-diffuse.

\section{Discussion}

The present study demonstrated that thyroid lesion showing hypothyroidism was closely associated with IgG4-related disease on the basis that 1) a high prevalence of 
hypothyroidism (19\%) was evident in patients with IgG4-related disease, 2) an active state of IgG4-related disease was observed in hypothyroidism patients based on significantly abnormal values of activity markers, including IgG4, CIC, and C3, 3) thyroid CT images of patients with clinical hypothyroidism revealed a significantly greater volume and generally lower density compared with normal subjects, euthyroid patients, and patients with subclinical hypothyroidism, 4) corticosteroid therapy ameliorated thyroid function in IgG4-related disease hypothyroidism similarly to other types of IgG4-related disease, and 5) focal histological evidence of IgG4-related disease in the non-cancerous thyroid tissue of one patient demonstrated infiltration of lymphocytes and IgG4-bearing plasma cells and loss of thyroid follicles. Together, these findings support our contention of a new disease concept termed IgG4-related thyroiditis.

The prevalence of hypothyroidism has been found to be significantly elevated in patients with IgG4-related disease. The present study showed this condition in $19 \%$ (22/114) of patients with IgG4-related disease, and a previous study performed by our group revealed that $27 \%$ of patients with AIP had hypothyroidism.[16] Hypothyroidism 
was also found in $18 \%$ of patients with histologically confirmed lymphoplasmacytic sclerosing pancreatitis, which is the histological counterpart of AIP,[23] and another study found clinical hypothyroidism requiring levothyroxine supplementation in $14.4 \%$ (14/97) of patients with AIP.[17] Thus, an estimated 14-28\% of patients with IgG4-related disease that includes AIP have hypothyroidism. Conversely, our previous study showed that none of 41 patients with chronic pancreatitis had hypothyroidism,[16] and the prevalence of this disorder in the general population was reported to be $4.6 \%$ in the National Health and Nutrition Examination Survey III.[24] These findings support the notion that thyroid lesion showing hypothyroidism is a complication of IgG4-related disease with a relatively high prevalence.

In our cohort, serum markers associated with IgG4-related disease indicated an active state. In other forms of IgG4-related disease, serum concentrations of IgG, IgG4, $\beta 2 \mathrm{MG}$, and sIL2R were initially high, decreased sharply after corticosteroid therapy in parallel with amelioration of clinical symptoms, and then increased during relapse of highly active state, indicating that these serum markers accurately reflect the activity of the disease.[3, 19, 22] Serum concentrations of compliment C3 and C4 have also been 
decreased in patients with AIP and IgG4-related nephritis with high CIC values, [25, 26]

thus representing other possible activity markers for IgG4-related disease. The present

study showed that levels of the above markers were significantly altered in the hypothyroid state of IgG4-related disease compared with those of euthyroid state, indicating that hypothyroidism represented a highly active state of IgG4-related disease and was closely associated with its development and pathophysiology. Moreover, we found a similar prevalence of complications with other IgG4-related diseases in the hypothyroidism and euthyroid state groups, as is characteristic of this disorder.

Typical imaging findings of active state IgG4-related disease include swelling of affected organs, such as in AIP [1, 2] and Mikulicz's disease.[19] We observed that thyroid CT images of clinical hypothyroidism had a significantly greater volume compared with those of normal subjects, euthyroid patients, and patients with subclinical hypothyroidism, although no patients showed marked goiter. It is possible that elevation of TSH stimulates thyroid-follicular growth, resulting in thyroid enlargement; however, our patients with clinical hypothyroidism showed a generally lower thyroid density compared with the other groups, and alteration of CT density is 
not dependent on TSH stimulation. Taken together, these results suggest that thyroid lesion of clinical hypothyroidism presents with similar imaging findings to other types of IgG4-related disease and indicate identical inflammatory processes and tissue damage.

Pathological analysis of thyroid tissue samples from a patient with IgG4-related disease whose thyroid was resected based on the diagnosis of thyroid cancer showed focal infiltration of lymphocytes and IgG4-bearing plasma cells and atrophic loss of thyroid follicles, suggesting that inflammation and tissue damage occur in the thyroid gland of IgG4-related disease even in the euthyroid state. In patients with clinical hypothyroidism, the inflammatory process may diffusely involve the thyroid tissue, although we were only able to examine the thyroid tissues of normal thyroid functions. In another patient with hypothyroidism, long-term serial thyroid image changes showed progressive atrophy and density loss, which raised the possibility that thyroid lesion with persistent IgG4-related inflammation may cause tissue atrophy in a manner comparable to chronic stage AIP.[22]

Hypothyroidism in IgG4-related disease responds favorably to corticosteroid 
therapy, which is a cardinal feature of IgG4-related disease,[1, 3, 19] and treatment outcome has been applied to various diagnostic criteria for this condition, including AIP.[18, 27, 28] However, the suppressive effect of corticosteroids on TSH expression may have affected hormone levels in such cases. The present study disclosed that free T4 levels in patients with IgG4-related disease hypothyroidism increased significantly after corticosteroid therapy, indicating that treatment may improve thyroid function through a suppressive effect on immune reactions and the inflammatory process. These findings support the notion that hypothyroidism is a closely associated lesion of IgG4-related disease.

In order further establish the entity of IgG4-related thyroiditis, we compared IgG4-related thyroid lesions with other thyroid diseases, such as autoimmune chronic thyroiditis (Hashimoto's thyroiditis) and Riedel's thyroiditis. Although IgG4-related thyroid lesion has been diagnosed with or included in Hashimoto's thyroiditis, IgG4-related thyroiditis seemed to represent an entity distinct from Hashimoto's thyroiditis in our study since 1) patients with IgG4-related thyroid lesion were older and showed a significant male preponderance, as seen in AIP and other forms of 
IgG4-related disease, 2) few patients with IgG4-related thyroid lesion showed significant diffuse goiter, 3) half of the IgG4-related hypothyroidism group had no detectable thyroid autoantibodies, and 4) patients with IgG4-related hypothyroidism showed a favorable response to corticosteroid therapy. Moreover, Hashimoto's thyroiditis generally shows diffuse goiter and few cases display atrophy.[29][29] A previous report demonstrated that Hashimoto's thyroiditis could be classified into two groups based on immunostaining for IgG4: IgG4 thyroiditis (IgG4-positive plasma cell-rich thyroiditis) and non-IgG4 thyroiditis (IgG4-positive plasma cell-poor thyroiditis).[15] IgG4 thyroiditis presented with severe lymphoplasmacytic infiltration, dense fibrosis, marked follicular cell degeneration, oxyphilic change, and lymphoid follicle formation, and was considered to be very close to the fibrous variant of Hashimoto's autoimmune thyroiditis[30] by presenting a clinicopathological picture distinct from that of typical Hashimoto's disease. Accordingly, IgG4-related thyroiditis may share clinical features with the variant form of Hashimoto's autoimmune thyroiditis, although we were not able to confirm these pathological findings.

Lastly, it is known that MIF, which is considered to be identical to IgG4-related 
disease, is complicated with Riedel's thyroiditis. A previous report showed that thyroidectomy samples of Riedel's thyroiditis stained positively for IgG4-bearing plasma cells.[14] However, we did not find severe fibrosis invading the surrounding organs in our patients with IgG4-related disease. Although we cannot completely exclude Riedel's thyroiditis in our cohort, further study of large groups of patients with this condition is needed to clarify whether it can also be included as an IgG4-related disease with respect to clinical features, serum IgG4 concentration, pathological findings, systemic involvement, and corticosteroid response.

In conclusion, based characteristic clinical, serologic, imaging, and pathological findings, IgG4-related disease is demonstrably complicated with hypothyroidism or thyroid lesion, which supports the proposal of a new disease entity termed IgG4-related thyroiditis. Awareness of IgG4-related thyroiditis will lead to appropriate corticosteroid treatment and may prevent progression to a fibrous state. 


\section{List of abbreviations}

AIP: autoimmune pancreatitis, CIC: circulating immune complex, sIL2R: soluble IL2 receptor, MIF: multifocal idiopathic fibrosclerosis, TSH: thyroid stimulating hormone, anti-TG: antithyroglobulin, anti-TPO: anti-thyroid peroxidase, ANA: antinuclear antibody, $\beta 2-\mathrm{MG}$ : $\beta 2$-microglobulin, CT: computed tomography.

\section{Acknowledgments}

This work was supported partially by the Research Program of Intractable Disease provided by the Ministry of Health, Labor, and Welfare of Japan, and in part by Grants-in-aid for Scientific Research from the Ministry of Education, Science, Sports, and Culture of Japan (23591012). The authors greatly appreciate Dr. Kayoko Higuchi and Dr. Akihiko Yoshizawa for their support in pathological analysis and Trevor Ralph for his English editorial assistance. 


\section{References}

1. Kawa S, Hamano H, Kiyosawa K. Pancreatitis. St Louis: Academic Press, 2006.

2. Kawa S, Fujinaga Y, Ota M, Hamano H, Bahram S. Autoimmune Pancreatitis and Diagnostic Criteria. Current Immunology Reviews 2011;7: 144-61.

3. Hamano H, Kawa S, Horiuchi A, Unno H, Furuya N, Akamatsu T et al. High serum IgG4 concentrations in patients with sclerosing pancreatitis. New England Journal of Medicine 2001;344 10: 732-8.

4. Hamano H, Kawa S, Ochi Y, Unno H, Shiba N, Wajiki M et al. Hydronephrosis associated with retroperitoneal fibrosis and sclerosing pancreatitis. Lancet 2002;359 9315: 1403-4.

5. Hamano H, Arakura N, Muraki T, Ozaki Y, Kiyosawa K, Kawa S. Prevalence and distribution of extrapancreatic lesions complicating autoimmune pancreatitis. J Gastroenterol 2006;41 12: 1197-205.

6. Fujinaga Y, Kadoya M, Kawa S, Hamano H, Ueda K, Momose M et al. Characteristic findings in images of extra-pancreatic lesions associated with autoimmune pancreatitis. Eur J Radiol 2009;76 2: 228-38.

7. Kawa S, Okazaki K, Kamisawa T, Shimosegawa T, Tanaka M. Japanese consensus guidelines for management of autoimmune pancreatitis: II. Extrapancreatic lesions, differential diagnosis. J Gastroenterol 2010;45: 1264-71.

8. Stone JH, Zen Y, Deshpande V. IgG4-related disease. N Engl J Med 2012;366 6: 539-51.

9. Kawa S, Sugai S. History of autoimmune pancreatitis and Mikulicz's disease. Current Immunology Review 2011;7 2: 137-43.

10. Comings DE, Skubi KB, Van Eyes J, Motulsky AG. Familial multifocal fibrosclerosis. Findings suggesting that retroperitoneal fibrosis, mediastinal fibrosis, sclerosing cholangitis, Riedel's thyroiditis, and pseudotumor of the orbit may be different manifestations of a single disease. Ann Intern Med 1967;66 5: 884-92.

11. Clark A, Zeman RK, Choyke PL, White EM, Burrell MI, Grant EG et al. Pancreatic pseudotumors associated with multifocal idiopathic fibrosclerosis. Gastrointest Radiol 1988;13 1: 30-2. 
12. Kawaguchi K, Koike M, Tsuruta K, Okamoto A, Tabata I, Fujita N.

Lymphoplasmacytic sclerosing pancreatitis with cholangitis: a variant of primary sclerosing cholangitis extensively involving pancreas. Hum Pathol 1991;22 4: 387-95.

13. Kamisawa T, Funata N, Hayashi Y, Tsuruta K, Okamoto A, Amemiya K et al. Close relationship between autoimmune pancreatitis and multifocal fibrosclerosis. Gut 2003;52 5: 683-7.

14. Dahlgren M, Khosroshahi A, Nielsen GP, Deshpande V, Stone JH. Riedel's thyroiditis and multifocal fibrosclerosis are part of the IgG4-related systemic disease spectrum. Arthritis Care Res (Hoboken) 2010;62 9: 1312-8.

15. Li Y, Bai Y, Liu Z, Ozaki T, Taniguchi E, Mori I et al. Immunohistochemistry of IgG4 can help subclassify Hashimoto's autoimmune thyroiditis. Pathol Int 2009;59 9: 636-41.

16. Komatsu K, Hamano H, Ochi Y, Takayama M, Muraki T, Yoshizawa K et al. High prevalence of hypothyroidism in patients with autoimmune pancreatitis. Dig Dis Sci 2005;50 6: 1052-7.

17. Sah RP, Chari ST. Clinical hypothyroidism in autoimmune pancreatitis. Pancreas 2010;39 7: 1114-6.

18. Otsuki M, Chung JB, Okazaki K, Kim MH, Kamisawa T, Kawa S et al. Asian diagnostic criteria for autoimmune pancreatitis: consensus of the Japan-Korea Symposium on Autoimmune Pancreatitis. J Gastroenterol 2008;43 6: 403-8.

19. Yamamoto M, Takahashi H, Shinomura Y. Mikulicz's Disease and its Extraglandular Lesions. Current Immunology Reviews 2011;7 2: 162-71.

20. Ghazale A, Chari ST, Zhang L, Smyrk TC, Takahashi N, Levy MJ et al. Immunoglobulin G4-associated cholangitis: clinical profile and response to therapy. Gastroenterology 2008;134 3: 706-15.

21. Umehara H, Okazaki K, Masaki Y, Kawano M, Yamamoto M, Saeki T et al. Comprehensive diagnostic criteria for IgG4-related disease (IgG4-RD), 2011. Mod Rheumatol 2012;22 1: 21-30.

22. Kawa S, Hamano H, Ozaki Y, Ito T, Kodama R, Chou Y et al. Long-term follow-up of autoimmune pancreatitis: characteristics of chronic disease and recurrence. Clin Gastroenterol Hepatol 2009;7 11 Suppl: S18-22.

23. Abraham SC, Wilentz RE, Yeo CJ, Sohn TA, Cameron JL, Boitnott JK et al. 
Pancreaticoduodenectomy (Whipple resections) in patients without malignancy: are they all 'chronic pancreatitis'? Am J Surg Pathol 2003;27 1: 110-20.

24. Hollowell JG, Staehling NW, Flanders WD, Hannon WH, Gunter EW, Spencer CA et al. Serum TSH, T(4), and thyroid antibodies in the United States population (1988 to 1994): National Health and Nutrition Examination Survey (NHANES III). J Clin Endocrinol Metab 2002;87 2: 489-99.

25. Muraki T, Hamano H, Ochi Y, Komatsu K, Komiyama Y, Arakura N et al. Autoimmune pancreatitis and complement activation system. Pancreas 2006;32 1: $16-21$.

26. Saeki T, Nishi S, Imai N, Ito T, Yamazaki H, Kawano M et al. Clinicopathological characteristics of patients with IgG4-related tubulointerstitial nephritis. Kidney Int 2010.

27. Chari ST, Smyrk TC, Levy MJ, Topazian MD, Takahashi N, Zhang L et al. Diagnosis of autoimmune pancreatitis: the Mayo Clinic experience. Clin Gastroenterol Hepatol 2006;4 8: 1010-6; quiz 934.

28. Kim KP, Kim MH, Kim JC, Lee SS, Seo DW, Lee SK. Diagnostic criteria for autoimmune chronic pancreatitis revisited. World J Gastroenterol 2006;12 16: 2487-96.

29. Davies TF. Ord-Hashimoto's disease: renaming a common disorder--again. Thyroid 2003;13 4: 317.

30. Katz SM, Vickery AL, Jr. The fibrous variant of Hashimoto's thyroiditis. Hum Pathol 1974;5 2: 161-70. 
Table 1. Comparison of serum markers and imaging findings between hypothyroid state and euthyroid state patients with IgG4-related disease.

\begin{tabular}{|c|c|c|c|}
\hline & $\begin{array}{l}\text { Hypothyroid state } \\
\qquad(\mathrm{n}=22)\end{array}$ & $\begin{array}{l}\text { Euthyroid state } \\
\qquad(\mathrm{n}=92)\end{array}$ & $p^{\pi}$ \\
\hline Age & $69(46-80)$ & $66(38-85)$ & 0.386 \\
\hline $\operatorname{Sex}(M / F)$ & $19 / 3$ & $70 / 22$ & 0.447 \\
\hline Median (25-75\%) & & & $p^{\S}$ \\
\hline $\lg G$ & 3092 (1906-3659) & 2121(1647-2779) & 0.030 \\
\hline $\lg G 4$ & $852(472-1565)$ & $472(248-859)$ & 0.019 \\
\hline $\mathrm{CIC}$ & $10.6(5.1-20.9)$ & $5.6(3.6-9.6)$ & 0.019 \\
\hline$\beta 2 M G$ & $2.6(2.3-3.7)$ & $2.2(1.7-2.8)$ & 0.036 \\
\hline sIL2 receptor & $968(532-1306)$ & $869(601-1250)$ & 0.538 \\
\hline C3 & $85(57-98)$ & $105(80-127)$ & 0.022 \\
\hline C4 & $16.9(10.8-27.1)$ & $22.5(16.8-28.9)$ & 0.347 \\
\hline $\mathrm{CH} 50$ & $44.6(36.1-50.1)$ & $48.8(37.1-60.3)$ & 0.416 \\
\hline $\begin{array}{l}\text { Number of positive } \\
\text { patients }\end{array}$ & & & $\mathbf{p}^{\pi}$ \\
\hline Anti-TG Ab $(>10)$ & $10 / 20(50 \%)$ & $18 / 74(24 \%)$ & 0.026 \\
\hline Anti-TPO Ab (>10) & $4 / 19(21 \%)$ & $8 / 72(11 \%)$ & 0.448 \\
\hline Anti-TG Ab (>10) or & $10 / 20(50 \%)$ & $12 / 73(16 \%)$ & 0.002 \\
\hline \multicolumn{4}{|l|}{ Anti-TPO Ab (>10) } \\
\hline ANA $(\geq \times 80)$ & $7 / 19(37 \%)$ & $28 / 85$ (33\%) & 0.954 \\
\hline $\begin{array}{l}\text { Patients complicated } \\
\text { with } 2 \text { or more }\end{array}$ & $18 / 22(82 \%)$ & $68 / 92(74 \%)$ & 0.618 \\
\hline IgG4-related lesions & & & \\
\hline
\end{tabular}

$\S$ : Mann-Whitney U-test, I : Chi-square test 
Fig 1

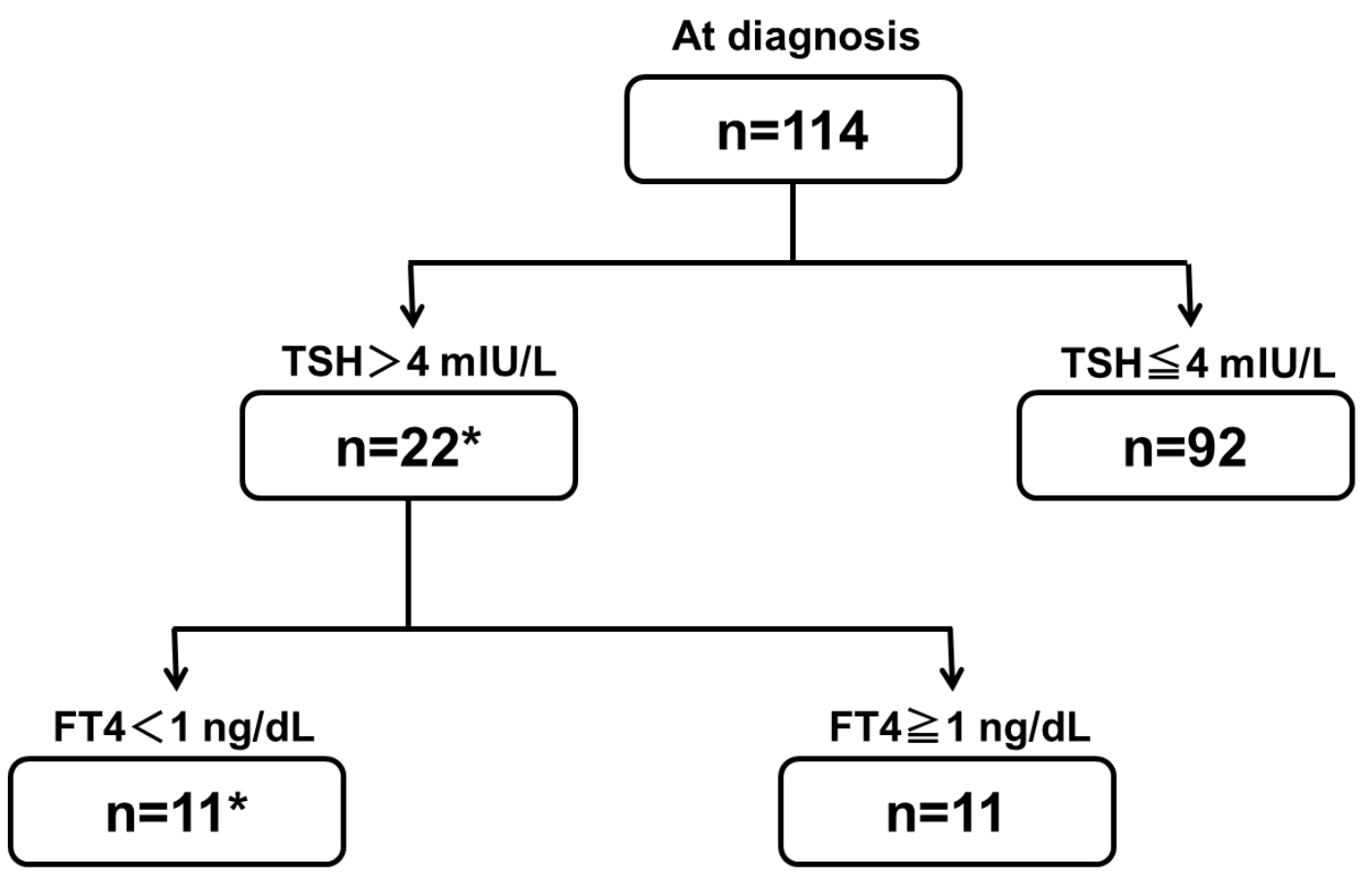


Fig 2
a) $\mathrm{TSH}(\mathrm{mlU} / \mathrm{L})$

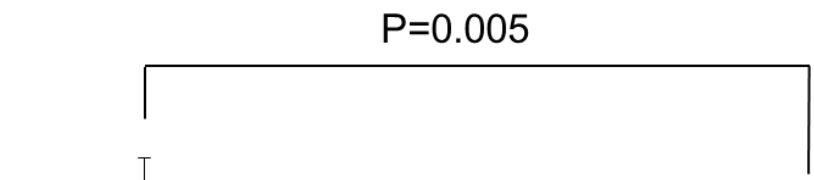

10

12

8

6

4

2

0

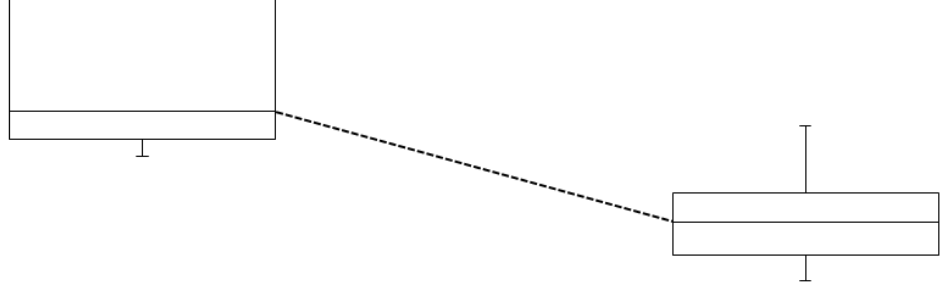

before

after

b) FT4 (ng/dL)

$\mathrm{P}=0.047$

1.6
1.2
0.8
0.4
0
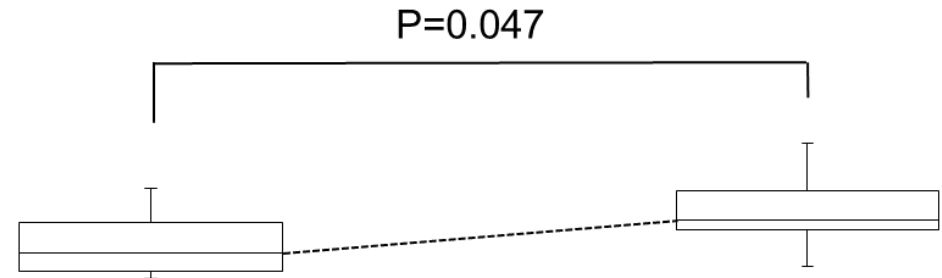

0

before

after 
Fig 3

a) Volume

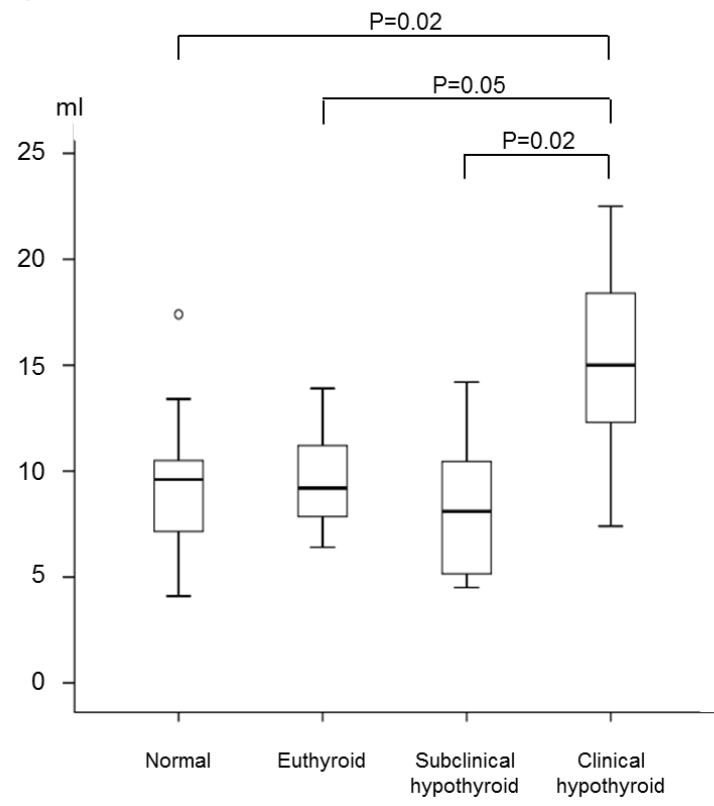

b) Density

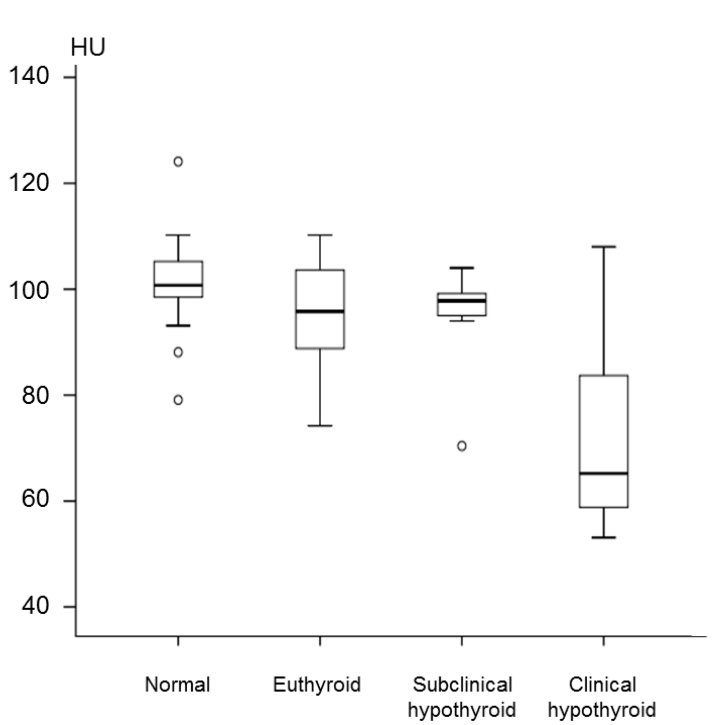


Fig 4
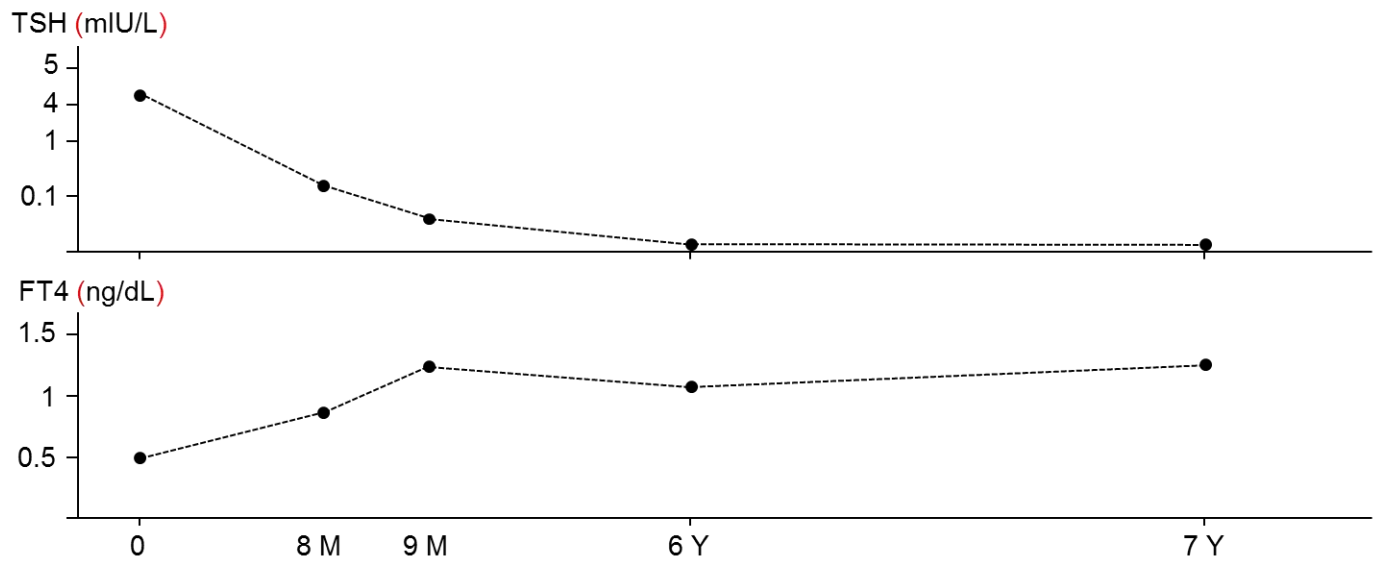

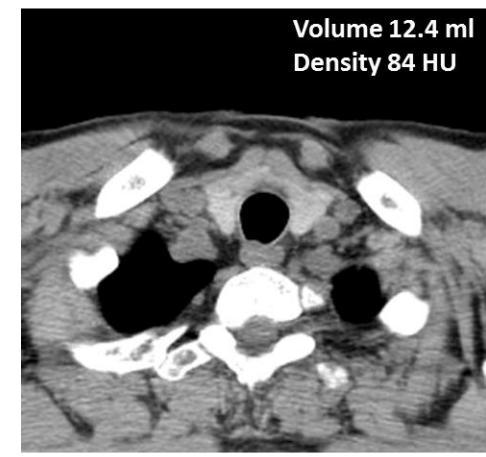

At diagnosis

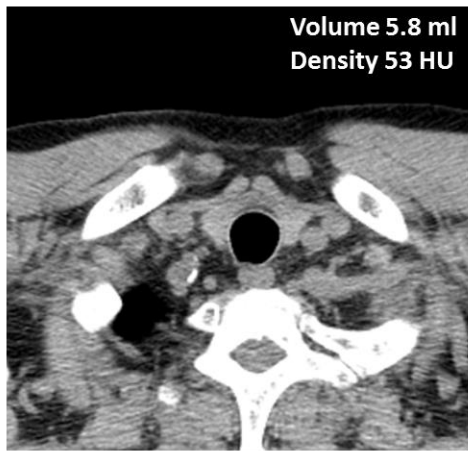

6 years later

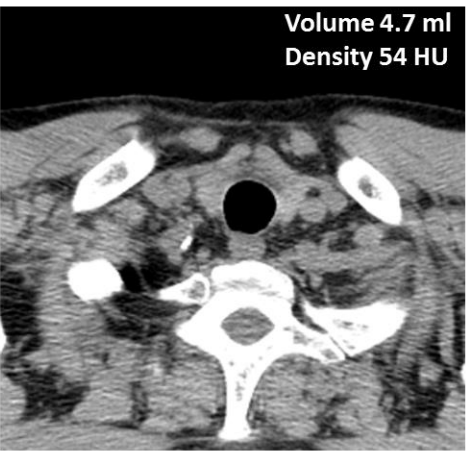

7 years later 
Fig 5
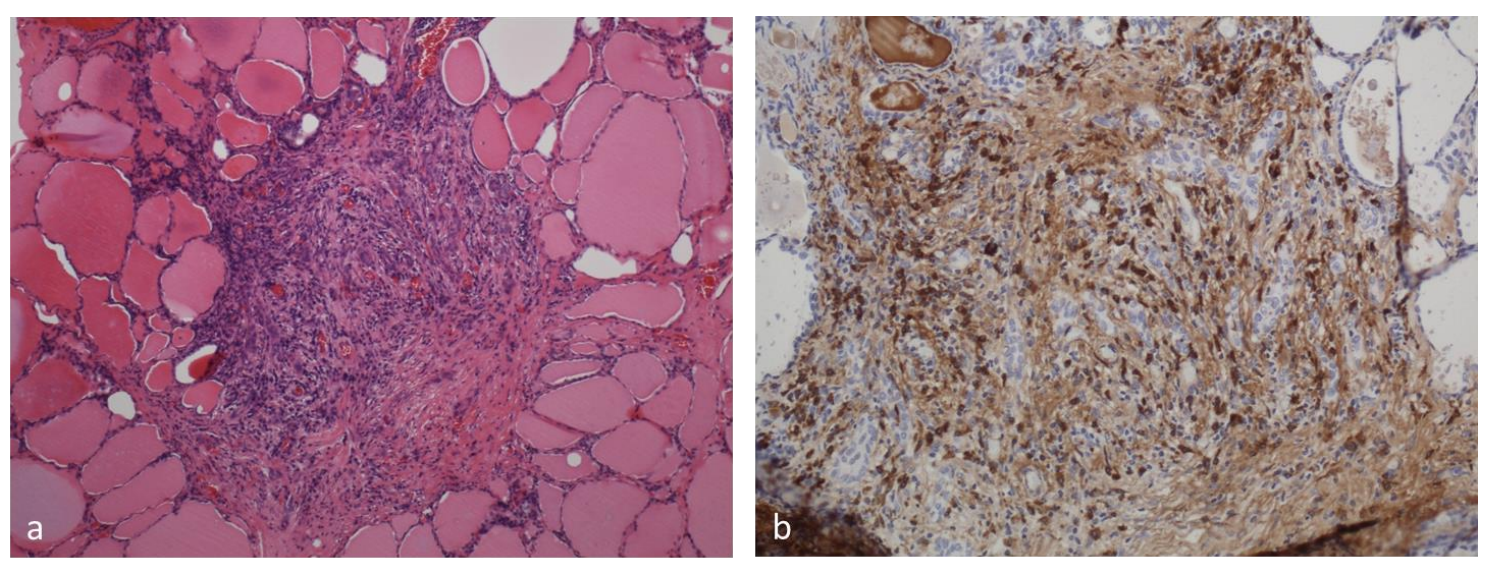


\section{Figure legends}

Figure 1. Frequency of hypothyroidism at diagnosis. * Two patients who required thyroxine supplementation were included in clinical hypothyroidism group.

Figure 2. Effect of predonisolone treatment on thyroid function with regard to TSH (a) and FT4 (b) in 10 patients with hypothyroidism (Wilcoxon test).

Figure 3. Comparison of thyroid gland volume (a) and density (b) (CT value) of the clinical hypothyroid group with those of the subclinical hypothyroid group, euthyroid group, and normal subjects.

Figure 4. A 61-year-old man with AIP had clinical hypothyroidism at diagnosis and was treated by corticosteroid and levothyroxine supplementation. During the clinical course, serial CT images of the thyroid gland showed progression of atrophy and low density.

Figure 5. Pathological analysis of non-cancerous thyroid tissue samples from a patient with IgG4-related disease and complicating thyroid cancer revealed prominent lymphoplasmacytic infiltration with loss of thyroid follicles (a) (HE staining) and IgG4-bearing plasma cells (b) (IgG4 immunostaining). 